\title{
Quantum Statistical Mechanics for Nonextensive Systems II
}

\author{
E. K. Lenzi ${ }^{1}$, R. S. Mendes ${ }^{2}$ and A. K. Rajagopal ${ }^{3}$ \\ ${ }^{1}$ Centro Brasileiro de Pesquisas Físicas, R. Dr. Xavier Sigaud 150, \\ 22290-180 Rio de Janeiro-RJ, Brazil \\ ${ }^{2}$ Departamento de Física, Universidade Estadual de Maringá, \\ Av. Colombo 5790, 87020-900 Maringá-PR, Brazil \\ ${ }^{3}$ Naval Research Laboratory, Washington DC 20375-5320
}

(August 12, 2018)

\begin{abstract}
In this paper, the Green function theory of quantum many-particle systems recently presented is reworked within the framework of nonextensive statistical mechanics with a new normalized $q$-expectation values. This reformulation introduces a renormalization of temperature of the earlier theory and a self-consistency condition. The linear response theory is also presented, along with its two-particle Green function version. Finally, a Boltzmann transport-like equation is also developed here.
\end{abstract}

PACS number(s): 05.70.Ce, 05.30.-d, 05.20.-y, 05.30.Ch

Typeset using REVTEX 


\section{INTRODUCTION}

In two recent papers [1,2] a Green function theory of nonextensive many-particle systems was developed. This work is here reformulated in terms of a new version [3] of the Tsallis ensemble involving normalized q-expectation values in the generalized context. This new version obtained in this way has the following desirable properties which the early versions lacked [4,5]: invariance of the ensemble with respect to uniform translation of the energy

spectrum, the expectation value of a $c$-number is the same as that $c$-number and finally, the preservation of the formal additive structure of an operator associated with two subsystems (e.g., energy). All these features entail the appearance of renormalized temperature parameter replacing that in the previous formalism along with a self-consistent condition. The purpose this paper is to revisit the general Green function theory presented in [1,2] in terms of the new framework. In Sec. II the one-particle Green function theory for both the Bose and Fermi systems along with explicit results for free-particle systems is given. These have implications to experiments as described before [1,2]. In Sec. III a generalization of the linear response theory [6] in the new framework is given and it is re-expressed in some special cases in terms of the two-particle Green-function. This is related to scattering cross section as before [1]2]. In Sec. IV a Boltzmann transport-like equation is, for the first time, derived, for arbitray $q$. The final Sec. V contains conclusions of this work.

\section{THE GREEN FUNCTION THEORY}

The Tsallis entropy $S_{q}=\left(1-\operatorname{Tr} \hat{\rho}^{q}\right) /(q-1), \operatorname{Tr} \hat{\rho}=1, \hat{\rho}$ the system density matrix is maximized subject to normalized $q$-expectations values $\langle\hat{H}\rangle_{q}=\tilde{U}_{q}=\operatorname{Tr} \hat{H} \hat{\rho}^{q} / \operatorname{Tr} \hat{\rho}^{q}$, $\langle\hat{N}\rangle_{q}=\tilde{N}_{q}=\operatorname{Tr} \hat{N} \hat{\rho}^{q} / \operatorname{Tr} \hat{\rho}^{q}$. A detailed discussion of this in relation to the earlier formulations may be found in [3]. To distinguish from our earlier work, a tilda over the corresponding expressions in the foregoing sequel is used. The new density matrix determined in this way 
is then

$$
\begin{aligned}
\hat{\tilde{\rho}} & =\exp _{q}(-\hat{X}(\tilde{\beta}, \mu)) / \tilde{Z}_{q}, \\
\tilde{Z}_{q} & =\operatorname{Tr}\left[\exp _{q}(-\hat{X}(\tilde{\beta}, \mu))\right],
\end{aligned}
$$

where

$$
\begin{aligned}
\exp _{q}(-\hat{X}(\tilde{\beta}, \mu)) & \equiv(1-(1-q) \hat{X}(\tilde{\beta}, \mu))^{1 /(1-q)}, \\
X(\tilde{\beta}, \mu) & =\tilde{\beta}\left(\left(\hat{H}-\tilde{U}_{q}\right)-\mu\left(\hat{N}-\tilde{N}_{q}\right)\right)
\end{aligned}
$$

with $\tilde{\beta}=\beta / c_{q}$, and $c_{q}=\operatorname{Tr} \hat{\tilde{\rho}}^{q}$. Here $\tilde{\beta}$ is the normalized temperature. $\beta$ and $\beta \mu$ are the Lagrange multipliers as before associated with the normalized $q$-expectation values given above. The constant $c_{q}$ is a function of all these quantities. There are two self-consistency conditions that follow from Eq.(代), because

$$
\left(\tilde{Z}_{q} \hat{\tilde{\rho}}\right)^{1-q}=\left[1-(1-q) \tilde{\beta}\left(\left(\hat{H}-\tilde{U}_{q}\right)-\mu\left(\hat{N}-\tilde{N}_{q}\right)\right)\right]
$$

from which it follows that

$$
c_{q}=\tilde{Z}_{q}^{1-q}
$$

By using the expression for $\hat{\tilde{\rho}}$ in Eq.(11) in the definition of $c_{q}$, another relation is derived:

$$
c_{q}=\operatorname{Tr}\left[\exp _{q}(-\hat{X}(\tilde{\beta}, \mu))\right]^{q} / \tilde{Z}_{q}^{q} .
$$

Using Eq.(4) in Eq.(5) an equivalent expression is obtained for $\tilde{Z}_{q}$ :

$$
\tilde{Z}_{q}=\operatorname{Tr}\left[\exp _{q}(-\hat{X}(\tilde{\beta}, \mu))\right]^{q} .
$$

Noting that $\exp _{q}(-\hat{X}(\tilde{\beta}, \mu)) \equiv\left[\exp _{q}(-\hat{X}(\tilde{\beta}, \mu))\right]^{q}\{1-(1-q) \hat{X}(\tilde{\beta}, \mu)\}$ and since $\operatorname{Tr}\left[\exp _{q}(-\hat{X}(\tilde{\beta}, \mu))\right]^{q} \hat{X} \equiv 0$ by definition, these two expression for $\tilde{Z}_{q}$ become a tautology. The expression for a general normalized $q$-expectation value is then

$$
\begin{aligned}
\langle\hat{A}\rangle_{q} & =\tilde{A}_{q}=\frac{\operatorname{Tr} \hat{A} \hat{\tilde{\rho}}^{q}}{\operatorname{Tr} \hat{\tilde{\rho}}^{q}} \\
& =\operatorname{Tr} \hat{A}\left[\exp _{q}(-\hat{X}(\tilde{\beta}, \mu))\right]^{q} / c_{q} \tilde{Z}_{q}^{q} \\
& =\frac{\operatorname{Tr}\left\{\hat{A}\left[\exp _{q}(-\hat{X}(\tilde{\beta}, \mu))\right]^{q}\right\}}{\operatorname{Tr}\left[\exp _{q}(-\hat{X}(\tilde{\beta}, \mu)]^{q}\right.}
\end{aligned}
$$




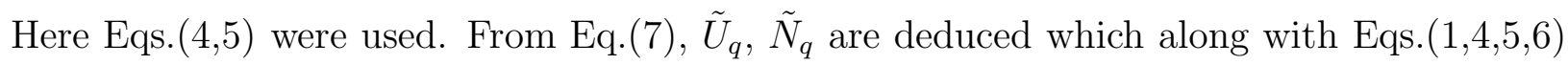
form the required self-consistent relations. In this form for the expectation value, only the "connected" diagrams appear and thus explain the results found [7,8] contract in to that obtained in [9] which did not used normalized $q$-expectation values. The one-particle Green function is then redefined as in [2] with the new normalized $q$-average and the density matrix given above:

$$
\begin{aligned}
\tilde{G}^{(q)}\left(1,1^{\prime} ; \beta, \mu\right) & =\frac{1}{i}\left\langle\mathrm{~T}\left(\Psi(1) \Psi^{\dagger}\left(1^{\prime}\right)\right)\right\rangle_{q}, \\
& \equiv \frac{1}{i} \frac{\operatorname{Tr}\left[\hat{\tilde{\rho}}^{q} \mathrm{~T}\left(\Psi(1) \Psi^{\dagger}\left(1^{\prime}\right)\right)\right]}{\operatorname{Tr} \hat{\tilde{\rho}}^{q}} .
\end{aligned}
$$

Using the contour integral representation employed in [1]:2]

$$
b^{1-z} \frac{i}{2 \pi} \int_{C} \mathrm{du} \exp (-u b)(-u)^{-z}=\frac{1}{\Gamma(z)}
$$

with $b>0$ and $\operatorname{Re} z>0$, where the contour $C$ starts from $+\infty$ on the real axis, encircles the origin once counterclockwise and returns to $+\infty$, Eq.(8) is expressed in terms of the Green function with $q=1$ :

$$
\tilde{G}^{(q)}\left(1,1^{\prime} ; \beta, \mu\right)=\int_{C} \mathrm{~d} u \tilde{K}_{q}^{(2)}(u) \tilde{Z}_{1}(-u(1-q) \tilde{\beta}, \mu) G^{(1)}\left(1,1^{\prime} ;-u(1-q) \tilde{\beta}, \mu\right)
$$

where

$$
\begin{aligned}
\tilde{K}_{q}^{(2)}(u) & =i \frac{\Gamma(1 /(1-q))}{2 \pi \tilde{Z}_{q}} \exp (-u)(-u)^{1 /(1-q)} \\
1 & =\int_{C} \mathrm{~d} u \tilde{K}_{q}^{(2)}(u) \tilde{Z}_{1}(-u(1-q) \tilde{\beta}, \mu) .
\end{aligned}
$$

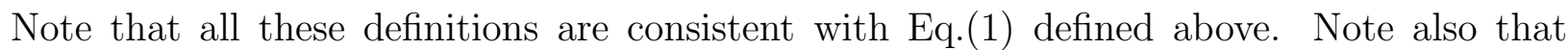
these expressions have similar appearance as those found in [2]. When $q=1$, the constant terms involving, $\tilde{U}_{q}, \tilde{N}_{q}$ factor out so that the one-particle Green function in integrand of Eq.(10) is the conventional extensive one: $\tilde{Z}_{1}$ appearing there however is the corresponding conventional partition function multiplied by $e^{-u \tilde{\beta}(1-q)\left(\tilde{U}_{q}-\mu \tilde{N}_{q}\right)}$. From Eq.(1) an equivalent expression is found for $\tilde{Z}_{q}$ : 


$$
\begin{aligned}
\tilde{Z}_{q} & =\int_{C} \mathrm{~d} u K_{q}^{(1)}(u) \tilde{Z}_{1}(-u(1-q) \tilde{\beta}, \mu) \\
K_{q}^{(1)} & =\frac{i \Gamma\left(\frac{2-q}{1-q}\right)}{2 \pi} \exp (-u)(-u)^{-(2-q) /(1-q)} \\
& =\frac{\tilde{Z}_{q}}{u(1-q)} \tilde{K}_{q}^{(2)}(u) .
\end{aligned}
$$

As in [2], correlation functions are introduced. Following KB [10], we introduce correlation functions

$$
\begin{aligned}
& \tilde{G}_{>}^{(q)}\left(11^{\prime} ; \beta, \mu\right)=\frac{1}{i}\left\langle\Psi(1) \Psi^{\dagger}\left(1^{\prime}\right)\right\rangle_{q} \\
& \tilde{G}_{<}^{(q)}\left(11^{\prime} ; \beta, \mu\right)=\frac{ \pm}{i}\left\langle\Psi^{\dagger}\left(1^{\prime}\right) \Psi(1)\right\rangle_{q} .
\end{aligned}
$$

The notation $>$ and $<$ is intended to exhibit the feature that $\tilde{G}^{(q)}\left(1,1^{\prime} ; \beta, \mu\right)=\tilde{G}_{>}^{(q)}\left(1,1^{\prime} ; \beta, \mu\right)$ for $t_{1}>t_{1^{\prime}}$ and $\tilde{G}^{(q)}\left(1,1^{\prime} ; \beta, \mu\right)=\tilde{G}_{<}^{(q)}\left(1,1^{\prime} ; \beta, \mu\right)$ for $t_{1}<t_{1^{\prime}}$. Using (9), we may similarly express $\tilde{G}_{>}^{(q)}$ and $\tilde{G}_{<}^{(q)}$ in terms of the corresponding grand canonical correlation functions. The spectral weight function in frequency space by taking the Fourier transform with respect to time differences, $A\left(\vec{r}_{1}, \vec{r}_{1^{\prime}} ; \omega\right)$, introduced in KB reflects only the properties of the Hamiltonian $\hat{H}$. The average occupation number in the grand canonical ensemble of a mode with energy $\omega, f(\omega, \beta)=(\exp (\beta(\omega-\mu)) \mp 1)^{-1}$, takes account of the basic permutation symmetry of the system. We can thus express $\tilde{G}_{>}^{(q)}$ and $\tilde{G}_{<}^{(q)}$ in terms of these in the following way:

$$
\begin{aligned}
& i \tilde{G}_{>}^{(q)}\left(\vec{r}_{1}, \vec{r}_{1^{\prime}} ; \omega ; \beta, \mu\right)=\int_{C} \mathrm{~d} u \tilde{K}_{q}^{(2)}(u) \tilde{Z}_{1}(-u(1-q) \tilde{\beta}, \mu) i \tilde{G}_{>}^{(1)}\left(\vec{r}_{1}, \vec{r}_{1^{\prime}} ; \omega ;-u(1-q) \tilde{\beta}, \mu\right) \\
= & \int_{C} \mathrm{~d} u \tilde{K}_{q}^{(2)}(u)(1 \pm f(\omega,-u(1-q) \tilde{\beta}, \mu)) A\left(\vec{r}_{1}, \vec{r}_{1^{\prime}} ; \omega\right) \tilde{Z}_{1}(-u(1-q) \tilde{\beta}, \mu) \\
& i \tilde{G}_{<}^{(q)}\left(\vec{r}_{1}, \vec{r}_{1^{\prime}} ; \omega ; \beta, \mu\right)=\int_{C} \mathrm{~d} u \tilde{K}_{q}^{(2)}(u) \tilde{Z}_{1}(-u(1-q) \tilde{\beta}, \mu) i G_{<}^{(1)}\left(\vec{r}_{1}, \vec{r}_{1^{\prime}} ; \omega ;-u(1-q) \tilde{\beta}, \mu\right) \\
= & \pm \int_{C} \mathrm{~d} u \tilde{K}_{q}^{(2)}(u) f(\omega,-u(1-q) \tilde{\beta}, \mu) A\left(\vec{r}_{1}, \vec{r}_{1^{\prime}} ; \omega\right) \tilde{Z}_{1}(-u(1-q) \tilde{\beta}, \mu) .
\end{aligned}
$$

Thus the spectral weight function is found to be

$$
\begin{aligned}
A\left(\vec{r}_{1}, \vec{r}_{1^{\prime}} ; \omega\right) & =i\left(\tilde{G}_{>}^{(q)}\left(\vec{r}_{1}, \vec{r}_{1^{\prime}} ; \omega ; \beta, \mu\right)-\tilde{G}_{<}^{(q)}\left(\vec{r}_{1}, \vec{r}_{1^{\prime}} ; \omega ; \beta, \mu\right)\right) \\
& =\int_{C} \mathrm{~d} u \tilde{K}_{q}^{(2)}(u) A\left(\vec{r}_{1}, \vec{r}_{1^{\prime}} ; \omega\right) \tilde{Z}_{1}(-u(1-q) \tilde{\beta}, \mu)
\end{aligned}
$$


From this we deduce an important sum rule

$$
i \int_{-\infty}^{\infty} \frac{\mathrm{d} \omega}{2 \pi}\left(\tilde{G}_{>}^{(q)}\left(\vec{r}_{1}, \vec{r}_{1^{\prime}} ; \omega ; \beta, \mu\right)-\tilde{G}_{<}^{(q)}\left(\vec{r}_{1}, \vec{r}_{1^{\prime}} ; \omega ; \beta, \mu\right)\right)=\delta\left(\vec{r}_{1}-\vec{r}_{1^{\prime}}\right)
$$

This is just an expression of the equal time CCR of the particle fields. The spectral function weight is a property of the given systems not dependent on the ensemble and the results have similar appearance as in [2] with the modifications noted here. Using the Fourier representations of the step functions involved in the time-ordered Green function in Eq.(8), an important result, generalizing the result found in [8] is deduced:

$$
\begin{gathered}
\tilde{G}^{(q)}\left(1,1^{\prime} ; \beta, \mu\right)=\int_{C} \mathrm{~d} u \tilde{K}_{q}^{(2)}(u) \tilde{Z}_{1}(-u(1-q) \tilde{\beta}, \mu) \int_{-\infty}^{\infty} \frac{\mathrm{d} \omega}{2 \pi} e^{-i \omega\left(t_{1}-t_{1^{\prime}}\right)} \times \\
\times \int_{-\infty}^{\infty} \frac{\mathrm{d} \omega^{\prime}}{2 \pi} A\left(\vec{r}_{1}, \vec{r}_{1^{\prime}} ; \omega^{\prime}\right)\left\{P\left(\frac{1}{\omega-\omega^{\prime}}\right)-i \pi \delta\left(\omega-\omega^{\prime}\right)\left[\tanh \left(\frac{-u}{2} \tilde{\beta}(1-q)(\omega-\mu)\right)\right]^{\mp 1}\right\} .
\end{gathered}
$$

Note that in the view of Eq.(16), only the delta function term in this equation depends explicity of $q$ as in Ref. [B]. So far we have discussed the one-particle properties. The above development is similarly extended to generalize the many-particle $q$-Green functions. Using the same notations as in KB, we have in general,

$$
\begin{aligned}
& \tilde{G}_{n}^{(q)}\left(12 \ldots n, 1^{\prime} 2^{\prime} \ldots n^{\prime} ; \beta, \mu\right)=\frac{1}{i^{n}}\left\langle T\left(\Psi(1) \Psi(2) \ldots \Psi(n) \Psi^{\dagger}\left(1^{\prime}\right) \Psi^{\dagger}\left(2^{\prime}\right) \ldots \Psi^{\dagger}\left(n^{\prime}\right)\right)\right\rangle_{q} \\
= & \int_{C} \mathrm{~d} u \tilde{K}_{q}^{(2)}(u) \tilde{Z}_{1}(-u(1-q) \tilde{\beta}, \mu) G_{n}^{(1)}\left(12 \ldots n, 1^{\prime} 2^{\prime} \ldots n^{\prime} ;-u(1-q) \tilde{\beta}, \mu\right) .
\end{aligned}
$$

For a many-particle system with a Hamiltonian containing one-body potential $V_{1}\left(\vec{r}_{1}\right)$ and instantaneous two-body interaction potential $V_{2}\left(\vec{r}_{1}, \vec{r}_{2}\right)$, which is symmetric under interchange of 1 and 2 :

$$
\begin{aligned}
\hat{H} & \left.=\int \mathrm{d} \vec{r} \frac{\nabla \Psi^{\dagger}(\vec{r}, t) \cdot \nabla \Psi(\vec{r}, t)}{2 m}+\int \mathrm{d} \vec{r} V_{1}(\vec{r}) \Psi^{\dagger}(\vec{r}, t) \Psi(\vec{r}, t)\right) \\
& +\frac{1}{2} \iint \mathrm{d} \vec{r} \mathrm{~d} \vec{r}^{\prime} \Psi^{\dagger}(\vec{r}, t) \Psi^{\dagger}\left(\vec{r}^{\prime}, t\right) V_{2}\left(\vec{r}, \vec{r}^{\prime}\right) \Psi\left(\vec{r}^{\prime}, t\right) \Psi(\vec{r}, t) .
\end{aligned}
$$

We can express $\tilde{U}_{q}$ in terms of the Green function following KB

$$
\tilde{U}_{q}=\left.\frac{ \pm i}{4} \int \mathrm{d} \vec{r}\left[i\left(\frac{\partial}{\partial t}-\frac{\partial}{\partial t^{\prime}}\right)+\frac{\nabla \cdot \nabla^{\prime}}{m}-V_{1}(r)-V_{1}\left(r^{\prime}\right)\right] \tilde{G}_{<}^{(q)}\left(\vec{r}, t ; \vec{r}^{\prime}, t^{\prime}\right)\right|_{\vec{r}^{\prime}=\vec{r}, t^{\prime}=t} .
$$

For the free particle case $V_{1}(r)=0$ as in Eqs.(23), (24) we have, 


$$
\frac{\tilde{U}_{q}}{V}=\int_{C} \mathrm{~d} u \tilde{K}_{q}^{(2)}(u) \int_{-\infty}^{\infty} \frac{\mathrm{d} \omega}{2 \pi} \int \frac{\mathrm{d}^{D} p}{(2 \pi)^{D}}\left(\frac{\omega+p^{2} / 2 m}{2}\right) \frac{\tilde{Z}_{1}(-u(1-q) \tilde{\beta}, \mu)}{\left(e^{-u(1-q) \tilde{\beta}(\omega-\mu)} \mp 1\right)} A(\vec{p} ; \omega) .
$$

For a uniform system, we can take Fourier transforns with respect to $\vec{r}_{1}-\vec{r}_{1^{\prime}}$ in Eq. (15) and express the one-particle momentum distribution function $\langle\hat{N}(\vec{p})\rangle_{q}$ in terms of the spectral weight function of the N-particle system.

$$
\tilde{N}(\vec{p})_{q}= \pm \int_{C} \mathrm{~d} u \tilde{K}_{q}^{(2)}(u) \int_{-\infty}^{\infty} \frac{\mathrm{d} \omega}{2 \pi} \frac{A(\vec{p} ; \omega) \tilde{Z}_{1}(-u(1-q) \tilde{\beta}, \mu)}{\left(e^{-u(1-q) \tilde{\beta}(\omega-\mu)} \mp 1\right)} .
$$

Similarly the one-particle frequency distribution function $\langle\hat{N}(\omega)\rangle_{q}$ is given by

$$
\tilde{N}(\omega)_{q}= \pm V \int_{C} \mathrm{~d} u \tilde{K}_{q}^{(2)}(u) \frac{\tilde{Z}_{1}(-u(1-q) \tilde{\beta}, \mu)}{\left(e^{-u(1-q) \tilde{\beta}(\omega-\mu)} \mp 1\right)} \int \frac{\mathrm{d}^{D} p}{(2 \pi)^{D}} A(\vec{p} ; \omega) .
$$

Here $V$ is the volume of the $D$ dimensional space in which the particles reside. The chemical potential is determined by the expression for the $q$-mean value of the total number operator $\hat{N}$

$$
\frac{\tilde{N}_{q}}{V}= \pm \int_{C} \mathrm{~d} u \tilde{K}_{q}^{(2)}(u) \int_{-\infty}^{\infty} \frac{\mathrm{d} \omega}{2 \pi} \int \frac{\mathrm{d}^{D} p}{(2 \pi)^{D}} \frac{\tilde{Z}_{1}(-u(1-q) \tilde{\beta}, \mu)}{\left(e^{-u(1-q) \tilde{\beta}(\omega-\mu)} \mp 1\right)} A(\vec{p} ; \omega) .
$$

Equation (23) will now be used to derive the one-particle $q$-momentum distribution function for a free fermi gas, where $A(\vec{p}, \omega)=2 \pi \delta\left(\omega-\epsilon_{p}\right), \epsilon_{p}$ being the one-particle energy. The known partition function for the free fermi gas in the Gibbs theory need in this expression is approximated in a simple form

$$
\ln Z_{1}(\beta, \mu)=V\left(\frac{m}{2 \pi \beta}\right)^{3 / 2} \sum_{l=1}^{\infty} \frac{(-1)^{(l+1)} e^{\beta \mu l}}{l^{5 / 2}} \cong V\left(\frac{m}{2 \pi \beta}\right)^{3 / 2} e^{\beta \mu},
$$

and hence also a further approximation resembling the fugacity expansion of the classical Maxwell gas,

$$
Z_{1}(\beta, \mu) \cong \sum_{l=0}^{\infty} \frac{1}{l !}\left[V\left(\frac{m}{2 \pi \beta}\right)^{3 / 2}\right]^{l} e^{\beta \mu l},
$$

and $1 /\left(e^{x}+1\right)=\sum_{J=1}^{\infty}(-1)^{J+1} e^{-J x}$ for $\left|e^{-x}\right|<1$, we have finally the result,

$$
\begin{aligned}
\frac{\tilde{N}(\vec{p})_{q}}{V} & \cong \frac{\Gamma\left(\frac{1}{1-q}\right)}{\tilde{Z}_{q}} \sum_{l=0}^{\infty} \frac{1}{l !}\left[V\left(\frac{m}{2 \pi \tilde{\beta}}\right)^{3 / 2}\right]^{l} \\
& \times \sum_{J=1}^{\infty} \frac{(-1)^{J+1}}{\Gamma\left(\frac{1}{1-q}+\frac{3 l}{2}\right)}\left\{1+(1-q) \tilde{\beta}\left(\tilde{U}_{q}-\mu \tilde{N}_{q}+\mu l-J\left(\epsilon_{p}-\mu\right)\right)\right\}^{q /(1-q)+3 l / 2}
\end{aligned}
$$


An approximate expression for the $q$-mean momentum distribution function in the first version of the Tsallis formulation [3] (where ordinary $q$-mean values were used) has been used in the literature [11]. We can deduce such an expression as in [11] from the above by making a further drastic approximation by taking $l=0, \tilde{Z}_{q}=1$, dropping $\tilde{U}_{q}, \tilde{N}_{q}$ and $\tilde{\beta}=\beta$ to be in conformity with the first version of the Tsallis formalism [3], we obtain the result,

$$
\frac{\tilde{N}(\vec{p})_{q}}{V} \approx \sum_{J=1}^{\infty}(-1)^{J+1}\left\{1-(1-q) \beta J\left(\epsilon_{p}-\mu\right)\right\}^{q /(1-q)} .
$$

A futher approximation corresponding to the high temperature scheme where $\beta\left(\epsilon_{p}-\mu\right)<<$ 1 , we have

$$
\frac{\tilde{N}(\vec{p})_{q}}{V} \approx \sum_{J=1}^{\infty}(-1)^{J+1}\left\{1-(1-q) \beta\left(\epsilon_{p}-\mu\right)\right\}^{q J /(1-q)}=\frac{1}{1+\left\{1-(1-q) \beta\left(\epsilon_{p}-\mu\right)\right\}^{-q /(1-q)}} .
$$

In order to assess the accuracy of the approximation in Eq.(30) in relation to the more exact result in Eq.(29), we compare the respective second order terms of the series, ( $q J(q J-$ $(1-q)) / 2)\left(\beta\left(\epsilon_{p}-\mu\right)\right)^{2}$ and $(q(2 q-1) / 2)\left(J \beta\left(\epsilon_{p}-\mu\right)\right)^{2}$, and we find the error involved is of order $(1-q)$. This result was essentially obtained in [11] via a different approach to this problem. Similar calculations for the Bose and the Maxwell gases may be made on the same lines as above yielding corresponding expressions for the respective momentum distribution functions.

\section{LINEAR RESPONSE FUNCTIONS}

The linear response is now calculated, following [6], using this new prescription:

$$
\langle\Delta \hat{B}(t)\rangle_{q} \equiv \operatorname{Tr}[\hat{P}(t) \hat{B}]-\operatorname{Tr}[\hat{\tilde{P}}(\hat{H} ; q, \tilde{\beta}) \hat{B}]
$$

where $\hat{P}(t)=\hat{\rho}^{q}(t) / \operatorname{Tr} \hat{\rho}^{q}(t)$. With this new definitions, all averages are calculated with proper unit normalization. The time-dependent $P$-operator in Eq.(31) obeys the usual equation of motion with time-dependent Hamiltonian $\hat{H}-\hat{A} X(t)$ 


$$
i \hbar \frac{\partial}{\partial t} \hat{P}(t)=[\hat{H}, \hat{P}(t)]-[\hat{A}, \hat{P}(t)] X(t)
$$

with the initial condition $\hat{P}(t=-\infty)=\hat{\tilde{P}}(\hat{H} ; q, \beta)$ given by Eq.(11). Taking the trace over both sides, we observe that $\operatorname{Tr} \hat{P}(t)=1$ for all times. The solution to this linear order in $X(t)$ is the found the standard procedures:

$$
\hat{P}(t) \approx \hat{\tilde{P}}(\hat{H} ; q, \beta)-\frac{1}{i \hbar} \int_{-\infty}^{t} \mathrm{~d} t e^{-i\left(t-t^{\prime}\right) \hat{H} / \hbar}[\hat{A}, \hat{\tilde{P}}(\hat{H} ; q, \tilde{\beta})] e^{i\left(t-t^{\prime}\right) \hat{H} / \hbar}
$$

Thus

$$
\begin{aligned}
\langle\Delta \hat{\tilde{B}}\rangle_{q} & =\int_{-\infty}^{t} \mathrm{~d} t \tilde{\phi}_{B A}^{(q)}\left(t-t^{\prime}\right) X\left(t^{\prime}\right) \\
\tilde{\phi}_{B A}^{(q)}\left(t-t^{\prime}\right) & =-\frac{1}{i \hbar} \operatorname{Tr}\{[\hat{A}, \hat{\tilde{P}}(\hat{H} ; q, \beta)] \hat{B}(t)\} \\
& =\frac{1}{i \hbar} \operatorname{Tr}\{[\hat{A}, \hat{B}(t)] \hat{\tilde{P}}(\hat{H} ; q, \beta)\} .
\end{aligned}
$$

It appears therefore all the results and conclusions of [6] hold with the new definition of mean values with the renormalization of the temperature and the self-consistency condition in Eq.(10,3).

We now turn our attention to rewriting the dynamic response and the scattering cross section in the $q$-formalism in terms of the integrals over the usual ones as was done above. Reformulating the result obtained in Ref. [6], the dynamic linear response of a quantity $\hat{B}$ to an external probe that generates $\hat{A}$ in the $q$-formalism is

$$
\tilde{\chi}_{B A}^{(q)}(\omega, \beta, \mu)=\lim _{\epsilon \rightarrow 0} \int_{0}^{\infty} \mathrm{dt} e^{-i \omega t-\epsilon t} \frac{1}{i} \tilde{\phi}_{B A}^{(q)}(t, \beta, \mu)
$$

This in terms of the integral representation, is:

$$
\tilde{\chi}_{B A}^{(q)}(\omega, \beta, \mu)=\int_{C} \mathrm{~d} u \tilde{K}_{q}^{(2)}(u) \tilde{Z}_{1}(-u(1-q) \tilde{\beta}, \mu) \chi_{B A}^{(1)}(\omega,-u(1-q) \tilde{\beta}, \mu)
$$

where $\chi_{B A}^{(1)}(\omega,-u(1-q) \tilde{\beta}, \mu)$ is the usual Kubo dynamical response function evaluated now at a temperature $-u(1-q) \tilde{\beta}$. Following Ref. [6] in the context above and rewriting the $q$-averages of the anticommutator and commutator expressions, we have: 


$$
\begin{aligned}
\tilde{\Psi}_{B A}^{(q)}(t, \beta, \mu) & =\frac{1}{2} \operatorname{Tr}[\hat{\tilde{P}}(\hat{H}, \hat{N} ; q, \beta, \mu)[\hat{A}(0) \hat{B}(t)+\hat{B}(t) \hat{A}(0)]] \\
& =\frac{1}{2} \int_{C} \mathrm{~d} u \tilde{K}_{q}^{(2)}(u) Z_{1}(-u(1-q) \tilde{\beta}, \mu) \Psi_{B A}^{(1)}(t,-u(1-q) \tilde{\beta}, \mu) \\
\tilde{\Phi}_{B A}^{(q)}(t, \beta) & =\lim _{\epsilon \rightarrow 0} \int_{t}^{\infty} \mathrm{d} t^{\prime} e^{-\epsilon t^{\prime}} \operatorname{Tr}[\hat{\tilde{P}}(\hat{H}, \hat{N} ; q, \beta, \mu)[\hat{A}(0), \hat{B}(t)]] \\
& =\int_{C} \mathrm{~d} u \tilde{K}_{q}^{(2)}(u) \tilde{Z}_{1}(-u(1-q) \tilde{\beta}, \mu) \Phi_{B A}^{(q=1)}\left(t^{\prime},-u(1-q) \tilde{\beta}, \mu\right) .
\end{aligned}
$$

The fluctuation-dissipation theorem due to Kubo [12 for the extensive case, $(q=1)$ is

$$
\Psi_{B A}^{(1)}(\omega, \beta, \mu)=E_{\beta}(\omega) \Phi_{B A}^{(1)}(\omega, \beta, \mu)
$$

with

$$
E_{\beta}(\omega)=\frac{\omega}{2} \operatorname{coth}\left(\frac{\beta \omega}{2}\right)
$$

Here we obtain

$$
\begin{aligned}
\tilde{\Psi}_{B A}^{(q)}(\omega, \beta, \mu) & =\frac{\omega}{4} \int_{C} \mathrm{~d} u \tilde{K}_{q}^{(2)}(u) \tilde{Z}_{1}(-u(1-q) \tilde{\beta}, \mu) \\
& \left.\times \operatorname{coth}(-u(1-q) \tilde{\beta}) \Phi_{B A}^{(1)}(\omega,-u(1-q) \tilde{\beta}, \mu)\right) .
\end{aligned}
$$

We now relate the scattering function defined for example in, Lovesey [13], in the $q$-formalism as

$$
\tilde{S}^{(q)}(\vec{k}, \omega, \beta)=\frac{1}{2 \pi} \int_{-\infty}^{\infty} \mathrm{dt} \exp (-i \omega t)\left\langle\hat{A}^{\dagger}(0) \hat{A}(t)\right\rangle_{q}^{(c)}
$$

where $\hat{A}$ is the operator which affects the change in the states of the system in a scattering process. Here the superscript $(c)$ denotes canonical ensemble instead of the grand canonical ensemble used earlier. This is equivalent formally to setting $\mu=0$ in the earlier development. Then, using our transformation, we express this scattering function in terms of the usual $q=1$ scattering function

$$
\tilde{S}^{(q)}(\vec{k}, \omega, \beta)=\int_{C} \mathrm{~d} u \tilde{K}_{q}^{(2)}(u) \tilde{Z}_{1}(-u(1-q) \tilde{\beta}) S^{(1)}(\vec{k}, \omega,-u(1-q) \tilde{\beta}) .
$$

From [6], by taking $\hat{B}=\hat{A}^{\dagger}$, we have that the imaginary part of the $q$-susceptibility, $\tilde{\chi}_{\hat{A}^{\dagger} \hat{A}}^{(q)}(\omega, \beta)$ can be expressed in terms of the $q=1$ scattering function 


$$
\begin{aligned}
& \operatorname{Im} \tilde{\chi}_{\hat{A}^{\dagger} \hat{A}}^{(q)}(\vec{k}, \omega, \beta)=\pi \int_{C} \operatorname{du} \tilde{K}_{q}^{(2)}(u) \tilde{Z}_{1}(-u(1-q) \tilde{\beta}) \times \\
& \times\left(1-\exp (-u(1-q) \tilde{\beta}) S^{(1)}(\vec{k}, \omega,-u(1-q) \tilde{\beta}) .\right.
\end{aligned}
$$

We have thus expressed the $q$-scattering function as well as the imaginary part of the associated $q$-susceptibility in terms of the parametric integrals over a kernel multiplied by the usual scattering function which now depends on this parameter as displayed above. It is possible to obtain other properties as in Ref. [6] to this new version of the Tsallis statistics.

\section{BOLTZMANN TRANSPORT-LIKE EQUATION}

There exists a class of disturbances which are not conveniently described by the usual equilibrium Green's function [10]. For example, the disturbance produced by the externally

applied force field, $\hat{F}(\vec{r}, t)=\nabla U(\vec{r}, t)$. And many interesting physical phenomena appear as response of systems to external disturbances of this kind, for example in an ordinary gas, a slowly varying $U(\vec{r}, t)$ produces sound waves. Thus, we expect that this feature will appear in the generalized $q$-ensemble theory as in $q=1$. It is interesting therefore to extend the usual Boltzmann equation to the generalized case to understand this in the nonextensive context. In our formalism, this disturbance (force) may be represented by,

$$
\hat{H}^{\prime}(t)=\int \mathrm{d}^{3} r \hat{n}(r, t) U(r, t)
$$

where $\hat{n}(r, t)=\Psi_{U}^{\dagger}(r, t) \Psi_{U}(r, t)$. Let us to obtain the colisionless Boltzmann equation from the Green functions given above using the Hartree approximation in the same spirit as KB. By using the Heisenberg notation we have that the $q$-expectation average is given by

$$
\langle\hat{X}(\mathbf{R}, t)\rangle_{U, q}=\frac{\sum_{i} \hat{\tilde{\rho}}_{i}^{q}\left\langle i, t_{0}\left|\hat{X}_{U}(\mathbf{R}, t)\right| i, t_{0}\right\rangle}{\sum_{i} \hat{\tilde{\rho}}_{i}^{q}}
$$

where

$$
\hat{X}_{U}(\mathbf{R}, t)=\mathcal{V}(t)^{-1} \hat{X}(\mathbf{R}, t) \mathcal{V}(t)
$$

with 


$$
\mathcal{V}(t)=\mathrm{T}\left\{\exp \left[-i \int_{t_{0}}^{t} \mathrm{~d} t^{\prime} \int \mathrm{d}^{3} r^{\prime} \hat{n}\left(r^{\prime}, t^{\prime}\right) U\left(r^{\prime}, t^{\prime}\right)\right]\right\}
$$

From the Eq.(13) we have that,

$$
\begin{gathered}
\tilde{G}_{<}^{(q)}\left(1,1^{\prime} ; U\right)= \pm \frac{1}{i}\left\langle\mathrm{~T}\left(\Psi_{U}^{\dagger}\left(1^{\prime}\right) \Psi_{U}(1)\right)\right\rangle_{q} \\
\tilde{G}^{(q)}\left(12,1^{\prime} 2^{\prime} ; U\right)=\left(\frac{1}{i}\right)^{2}\left\langle\mathrm{~T}\left(\Psi_{U}(1) \Psi_{U}(2) \Psi_{U}^{\dagger}(2) \Psi_{U}^{\dagger}(1)\right)\right\rangle_{q}
\end{gathered}
$$

In terms of these Green's functions we may describe the response of a system, initially in thermodynamic equilibrium, to an applied disturbance $U(\vec{r}, t)$. In the same way as above, the average density at point $\mathbf{R}, T$ is

$$
\begin{aligned}
\langle\hat{n}(\mathbf{R}, T)\rangle_{U, q} & =\left\langle\Psi_{U}^{\dagger}(\mathbf{R}, T) \Psi_{U}(\mathbf{R}, T)\right\rangle_{q} \\
& = \pm i \tilde{G}_{<}^{(q)}(\mathbf{R}, T, \mathbf{R}, T ; U)
\end{aligned}
$$

and the current at the same point is

$$
\langle\hat{J}(\mathbf{R}, T)\rangle_{U, q}=\left\{\frac{\nabla-\nabla^{\prime}}{2 m i}\left[ \pm i \tilde{G}_{<}^{(q)}\left(\mathbf{R}, T, \mathbf{R}^{\prime}, T ; U\right)\right]\right\}_{\mathbf{R}=\mathbf{R}^{\prime}}
$$

where the conservation laws for the number of particles, the energy and the momentum are preserved here as well as in the usual case, and we can use them for derivation of the sound propagation. Here we define $f_{q}(\mathbf{p}, \mathbf{R}, T)\left(\right.$ with $\mathbf{r}=\vec{r}_{1}-\vec{r}_{1^{\prime}}, \mathbf{R}=1 / 2\left(\vec{r}_{1}+\vec{r}_{1^{\prime}}\right) t=t_{1}-t_{1^{\prime}}$ and $\left.T=1 / 2\left(t_{1}+t_{1^{\prime}}\right)\right)$ as,

$$
\begin{aligned}
f_{q}(\mathbf{p}, \mathbf{R}, T) & =\int \frac{\mathrm{d} \omega}{2 \pi} \tilde{G}_{<}^{(q)}(\mathbf{p}, \omega, \mathbf{R}, T ; U) \\
& =\int \mathrm{d}^{3} r e^{-i \mathbf{p} \cdot \mathbf{r}}\left\langle\Psi_{U}^{\dagger}\left(\mathbf{R}-\frac{\mathbf{r}}{2}, T\right) \Psi_{U}\left(\mathbf{R}+\frac{\mathbf{r}}{2}, T\right)\right\rangle_{q}
\end{aligned}
$$

generalizing the original definition proposed by Wigner. As in the usual case $f_{q}(\mathbf{p}, \mathbf{R}, T)$ leads to the generalized $q$-particle density

$$
\int \frac{\mathrm{d}^{3} p}{(2 \pi)^{3}} f_{q}(\mathbf{p}, \mathbf{R}, T)=\left\langle\Psi_{U}^{\dagger}(\mathbf{R}, T) \Psi_{U}(\mathbf{R}, T)\right\rangle_{q}=\langle\hat{n}(\mathbf{R}, T)\rangle_{q}
$$

and the generalized $q$-particle current 


$$
\langle\hat{J}(\mathbf{R}, T)\rangle_{U, q}=\int \frac{\mathrm{d}^{3} p}{(2 \pi)^{3}} \frac{\mathbf{p}}{m} f_{q}(\mathbf{p}, \mathbf{R}, T)
$$

This identification above of the distribution function $f_{q}(\mathbf{p}, \mathbf{R}, T)$ will enable us to see the relationship between to Green's functions, transport equations, and the colisionless Boltzmann equation. Thus, to obtain the colisionless Boltzmann equation we use

$$
\begin{aligned}
& \left(i \frac{\partial}{\partial t_{1}}+\frac{\nabla_{1}^{2}}{2 m}-U(1)\right) \tilde{G}^{(q)}\left(1,1^{\prime} ; U\right)=\delta\left(1-1^{\prime}\right) \\
\pm & \iint \mathrm{d} t_{2} \mathrm{~d} r_{2} v\left(\vec{r}_{1}-\vec{r}_{2}\right) \delta\left(t_{1}-t_{2}\right) \tilde{G}^{(q)}\left(12,1^{\prime} 2^{+} ; U\right)
\end{aligned}
$$

and

$$
\tilde{G}^{(q)}\left(12,1^{\prime} 2^{\prime} ; U\right)=\tilde{G}^{(q)}\left(1,1^{\prime} ; U\right) \tilde{G}^{(q)}\left(2,2^{\prime} ; U\right)
$$

After some simplification, we obtain

$$
\left(i \frac{\partial}{\partial t_{1}}+\frac{\nabla_{1}^{2}}{2 m}-U_{e f f}(1)\right) \tilde{G}^{(q)}\left(1,1^{\prime} ; U\right)=\delta\left(1-1^{\prime}\right)
$$

where

$$
U_{e f f}(\mathbf{R}, T)=U(\mathbf{R}, T) \pm i \int \mathrm{d} \mathbf{R}^{\prime} v\left(\mathbf{R}-\mathbf{R}^{\prime}\right) \tilde{G}_{<}^{(q)}\left(\mathbf{R}^{\prime}, T ; \mathbf{R}^{\prime}, T\right)
$$

By taking the difference of two equations in the variables 1 and $1^{\prime}$, we find

$$
\left\{i\left(\frac{\partial}{\partial t_{1}}+\frac{\partial}{\partial t_{1^{\prime}}}\right)+\left(\nabla_{1}+\nabla_{1^{\prime}}\right) \cdot\left(\frac{\nabla_{1}-\nabla_{1^{\prime}}}{2 m}\right)-\left[U_{\text {eff }}(1)-U_{\text {eff }}\left(1^{\prime}\right)\right]\right\} \tilde{G}^{(q)}\left(1,1^{\prime} ; U\right)=0 \text {. }
$$

Considering now $t_{1^{\prime}}=t_{1}^{+}=T$ and expressing Eq.(60) in terms of, $\mathbf{r}=\vec{r}_{1}-\vec{r}_{1^{\prime}}, \mathbf{R}=$ $1 / 2\left(\vec{r}_{1}+\vec{r}_{1^{\prime}}\right)$ and using Eq.(53) we have that

$$
\begin{aligned}
& \pm\left\{\frac{\partial}{\partial T}+\frac{\nabla_{\mathbf{R}} \cdot \nabla_{\mathbf{r}}}{i m}-\frac{1}{i}\left[U_{e f f}\left(\mathbf{R}+\frac{\mathbf{r}}{2}, T\right)-U_{e f f}\left(\mathbf{R}-\frac{\mathbf{r}}{2}, T\right)\right]\right\} \\
& \times \int \frac{\mathrm{d}^{3} p^{\prime}}{(2 \pi)^{3}} e^{i \mathbf{p}^{\prime} \cdot \mathbf{r}} f_{q}\left(\mathbf{p}^{\prime}, \mathbf{R}, T\right)=0 .
\end{aligned}
$$

Now let us suppose that $U_{\text {eff }}(\mathbf{R}, T)$ varies slowly in $\mathbf{R}$, then we obtain after some calculation the collisionless Bolztmann equation 


$$
\left[\frac{\partial}{\partial T}+\frac{\mathbf{p}}{2 m} \cdot \nabla_{\mathbf{R}}-\nabla_{\mathbf{R}} U_{e f f}(\mathbf{R}, T) \cdot \nabla_{\mathbf{p}}\right] f_{q}(\mathbf{p}, \mathbf{R}, T)=0
$$

where

$$
U_{e f f}(\mathbf{R}, T)=U(\mathbf{R}, T)+\int \mathrm{d} \mathbf{R}^{\prime} v\left(\mathbf{R}-\mathbf{R}^{\prime}\right) \int \frac{\mathrm{d}^{3} p^{\prime}}{(2 \pi)^{3}} f_{q}\left(\mathbf{p}^{\prime}, \mathbf{R}^{\prime}, T\right)
$$

\section{SUMMARY AND CONCLUSIONS}

In this paper we have developed in detail the Green function theory for nonextensive systems based on the $q$-ensemble of Tsallis. By means of a contour representation, Eq.(9), we have made this theory resemble the usual one for extensive systems given by L. P. Kadanoff and G. Baym [10], for example, even though in actual practice, the results are very different, as exemplified by the representative results given in Sec. II for a variety of situations. Before this development, thermodynamic quantities for model systems were computed in the Tsallis ensemble as for example in Ref. [9, 14]. With the present work, we believe that the theory of many particle systems for the Tsallis ensemble has been considerably extended and placed on par with the conventional theory based on the Gibbsian ensemble, in that we have been able to compute response functions besides the thermodynamic quantities. The case of $q$ different from unity is expected to apply for long-range interacting Hamiltonian systems [3], among others. From a formal point of view, noninteracting and short-range interacting systems are mathematically well posed problems only for $q \leq 1$. In conclusion, we have here developed the formalism associated with Tsallis statistics for describing nonextensive many-particle systems by a suitable generalization of the corresponding Green function techniques, so commonly employed in such studies for extensive systems. As with the usual Green function theory, which has been traditionally successful in explaining experimental observations, the present work enables us to analyze future possible experimental work on nonextensive systems. We may add that other forms of the nonextensive entropy such as $S_{q}^{(a)}=S_{q} / c_{q}$, deduced from considerations of form invariance [15] of the statement of maximum entropy principle and the metric structure in quantum density matrix theory, 
many also be expressed in contour integral form. The work presented here thus admits of applications elsewhere.

\section{ACKNOWLEDGEMENTS}

AKR thanks the Office of Naval Research for partial support of this work. EKL acknowledges partial support from CNPq and PRONEX (Brazilian agencies). 


\section{REFERENCES}

[1] A. K. Rajagopal, R. S. Mendes and E. K. Lenzi, Phys. Rev.Lett. 80, 3907 (1998)

[2] E. K. Lenzi, R. S. Mendes and A. K. Rajagopal ,Phys. Rev. E 59, 1397 (1999).

[3] C. Tsallis, R. S. Mendes and A. R. Plastino, Physica A 261, 534 (1998).

[4] C. Tsallis, J. Stat. Phys. 52, 479 (1988); see also Chaos, Solitons and Fractals, 6, 539 (1995).

[5] E. M. F. Curado and C. Tsallis, J. Phys. A 24, L69 (1991); Errata: 24, 3187 (1991); 25, 1019 (1992); See http://tsallis.cat.cbpf.br/biblio.htm for a periodically updated bibliography on the subject.

[6] A. K. Rajagopal, Phys. Rev. Lett. 76, 3469 (1996).

[7] S. Abe, Thermodynamics limit and classical ideal gas in nonextensive statistical mechanics with normalized q-expectation values, preprint (1998).

[8] S. Abe, Thermal Green functions in nonextensive statistical mechanics, Eur. Phys. J. B. (1999) in press.

[9] A. R. Plastino and A. Plastino and C. Tsallis, J. Phys. A 27, 5707 (1994).

[10] L. P. Kadanoff and G. Baym, Quantum Statistical Mechanics, (W. A. Benjamin, Inc., New York, 1962).

[11] F. Büyükkilç, D. Demirhan, and A. Gülec, Phys. Lett. A 197, 209 (1995).

[12] R. Kubo, J. Phys. Soc. Jpn. 12, 570 (1957).

[13] S. W. Lovesey, Condensaded Matter Physics, (The Benjamin/Cummings Plublishing Company, Massachusetts, 1980).

[14] S. Curilef, Phys. Lett. A 218, 11 (1996); see also S. Curilef, Z. Phys. B 100, 433 (1996).

[15] A. K. Rajagopal and Sumiyoshi Abe, submitted for publication (1999). 